\title{
R Wave Extraction from ECG Signal using Modified Wavelet Transform
}

\author{
Syed Hassaan Ahmed ${ }^{1}$, Tahir Zaidi ${ }^{2}$, Nauman Razzaq ${ }^{3}$ and Abdul Rehman ${ }^{4}+$ \\ College of E\&ME, National University of Sciences and Technology, Islamabad, Pakistan \\ University of Wah, Wah Cantt , Pakistan
}

\begin{abstract}
Electro Cardiogram (ECG) is used to measure and diagnose electrical activity of heart. R peak detection from ECG signal is our main goal. It is the basic mark for identification of different arrhythmias. In this paper, $\mathrm{R}$ wave extraction is performed by using Wavelet Transform. The wavelet transform has risen over late years as an effective time- frequency analysis and it is efficiently analyze complex non stationary signals. In this research, $\mathrm{R}$ wave is extracted accurately then heart beat is analyzed by the detection of RR intervals. In this research, $\mathrm{R}$ peak is detected accurately by this type of approach. By accurate detection of $\mathrm{R}$ peak, cardiac diseases can easily be identified such as Sinus tachycardia, Sinus bradycardia, Supraventricular tachycardia (SVT), atrial fibrillation (AF), Ventricular tachycardia, Heart block and Sudden Cardiac Arrest (SCA).
\end{abstract}

Keywords: ECG signal, R-wave, QRS complex-R interval, wavelet transform, ECG Peak detection

\section{Introduction}

Electrocardiogram (ECG) represents the electrical movement of the heart demonstrating the contraction and Relaxation of heart muscle.ECG is the diagnostic tool for the identification of electrical activities of heart. R peak detection form ECG signal is responsible for its identification. If arrhythmias are not treated properly then it causes sudden cardiac death [1]-[2].

In the previous couple of decades few techniques are evolved for ECG analysis and arrhythmia detection to enhance its accuracy and sensitivity. These methods include Wavelet coefficient [3], Autoregressive Modelling [4], RBF Neural Networks [5], self-organizing map [6], and fuzzy c-means clustering techniques [7]. Figure1 shows the typical ECG waveform with R-R interval and basic waves such as P, Q, R, S, T and $\mathrm{U}[8]$.

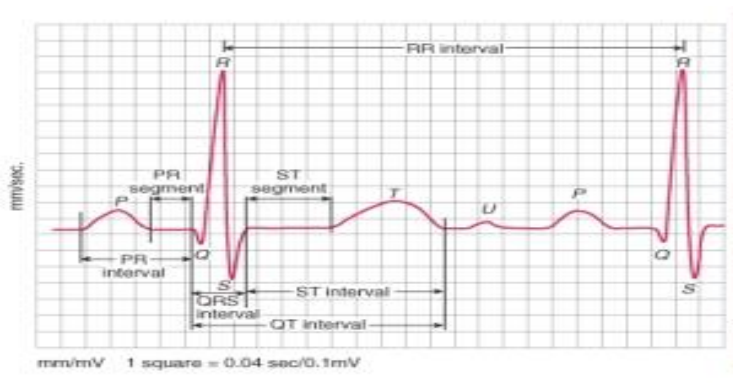

(a)

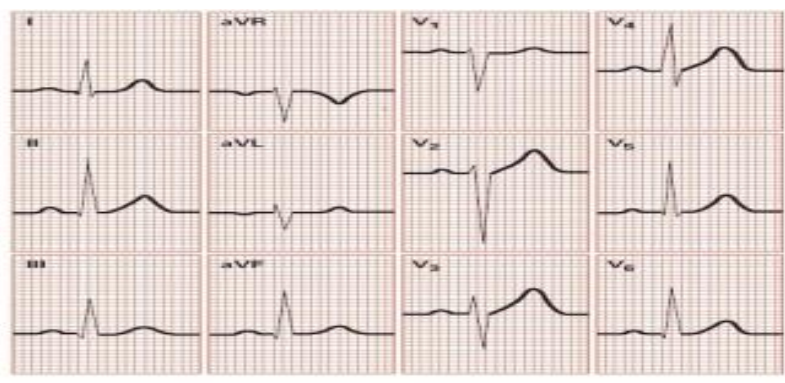

(b)

Fig. 1: ECG signal generation using MATLAB and its Real image

In the literature, ECG signal processors operate at different frequency ranging from $0.25 \mathrm{~Hz}$ to $400 \mathrm{kHz}$

\footnotetext{
+ Corresponding author. Tel.: + 92332-4742472.

E-mail address: Hassaanahmed01@ @otmail.com
} 
[9]-[12]. ECG Signal detection includes elimination of different noises like baseline drift [10]-[14], waveform detection [15]-[17], feature extraction [18], and heart rate classification [19]-[28]. Among the several techniques investigated in the literature are included time domain analysis [29], statistical approach [30]-[32], hybrid features [33], [34], frequency-based analysis [35], and time-frequency analysis [36]-[38] for feature extraction of ECG signals.

Heart Rate classification techniques were also used by several researchers, some of them have used waveform features extraction techniques [19]-[26] and some have used wavelet transform [23]-[24] method for its extraction. Wavelet analysis is used to eliminate noise from ECG signal and identify possible cardiovascular abnormalities. It is used for stationary as well as non-stationary signals. It gives both frequency and time domain information of signal during processing. In fact, it covers quite large area as it also deals with continuous and discrete domain signals.

\section{The Continuous Wavelet Transform}

A wavelet is simply a small wave has energy concentration in time for the analysis of transient, non stationary or time-varying phenomena as shown in Fig. 2.

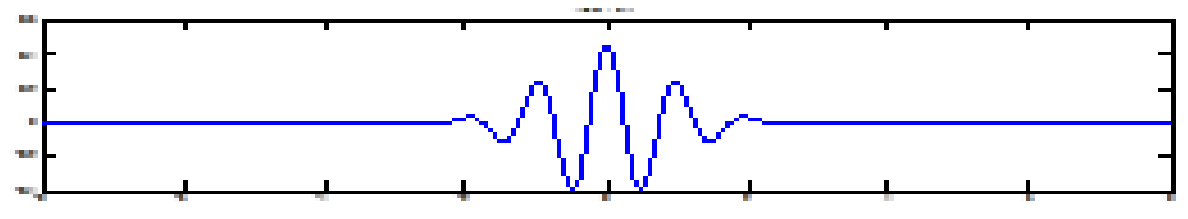

Fig. 2: Mother wavelet using continuous wavelet transform

Equation (1) highlights the continuous wavelet transform [39].

$$
W(\alpha, \beta)=\int_{-\infty}^{\infty} x(t) \frac{1}{\sqrt{|\alpha|}} \Psi^{*}\left(\frac{t-\beta}{\alpha}\right) d t
$$

\section{The Discrete Wavelet Transform}

The Continuous Wavelet Transform is highly redundant. The basic analytical expression for the Discrete Time Wavelet Transform (DWT) is expressed in equation (2).

$$
x(t)=\sum_{i=-\infty}^{\infty} \sum_{j=-\infty}^{\infty} d(i, j) 2^{-i / 2} \Psi\left(2^{i} t-j\right)
$$

Here scaling function is used to compute the DWT. Scaling function is defined by equation (3).

$$
\varphi(t)=\sum_{n=-\infty}^{\infty} \sqrt{2} c(n) \varphi(2 t-n)
$$

In the DWT, the wavelet can be defined from the scaling function as defined in equation (4).

$$
\Psi(t)=\sum_{n=-\infty}^{\infty} \sqrt{2} d(n) \varphi(2 t-n)
$$

\section{Methodology}

Flow Chart for R-wave detection using Wavelet Transform is demonstrated in Fig.3

\section{Results}

Fig 3 shows the implementation of Wavelet Transform algorithm for R-wave detection and shows the original Real Time ECG signal generation using MATLAB. Here Low pass and High Pass filter is applied to filter baseline wander and muscle noise and then Filtered signal is passed through Band Pass Filter. Derivative filter is applied on Filtered signal to highlight the QRS complex for the detection of R-wave[40]. Derivative signal is passed through moving Average filter. 
After taking its square to further removes the remaining noise. And Then Threshold Filter is applied to find Peaks of R-wave from ECG signal [13] and R-R interval is determined. Fig describes the R-wave detection from ECG signal using Wavelet Transform algorithm [41-44]. And it describes the R-wave detection using MATLAB and then Heart rate is calculated from R-R interval as by following Equation (5) as shown in Fig 4.

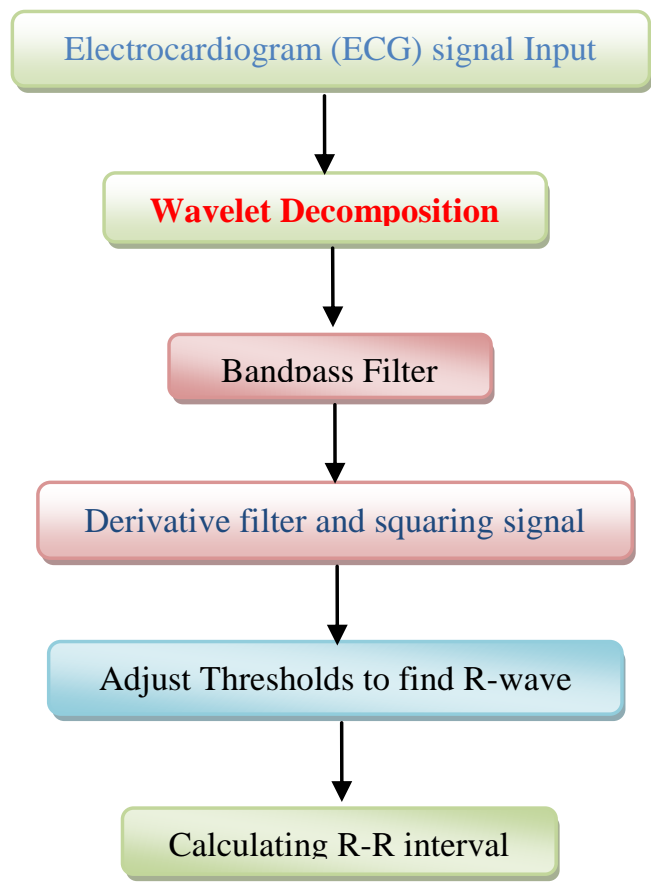

Fig. 3: Flow chart of our proposed model

Heart Rate $(B P M)=\frac{60 * \text { Sampling Rate }}{R-R \text { interval }}$
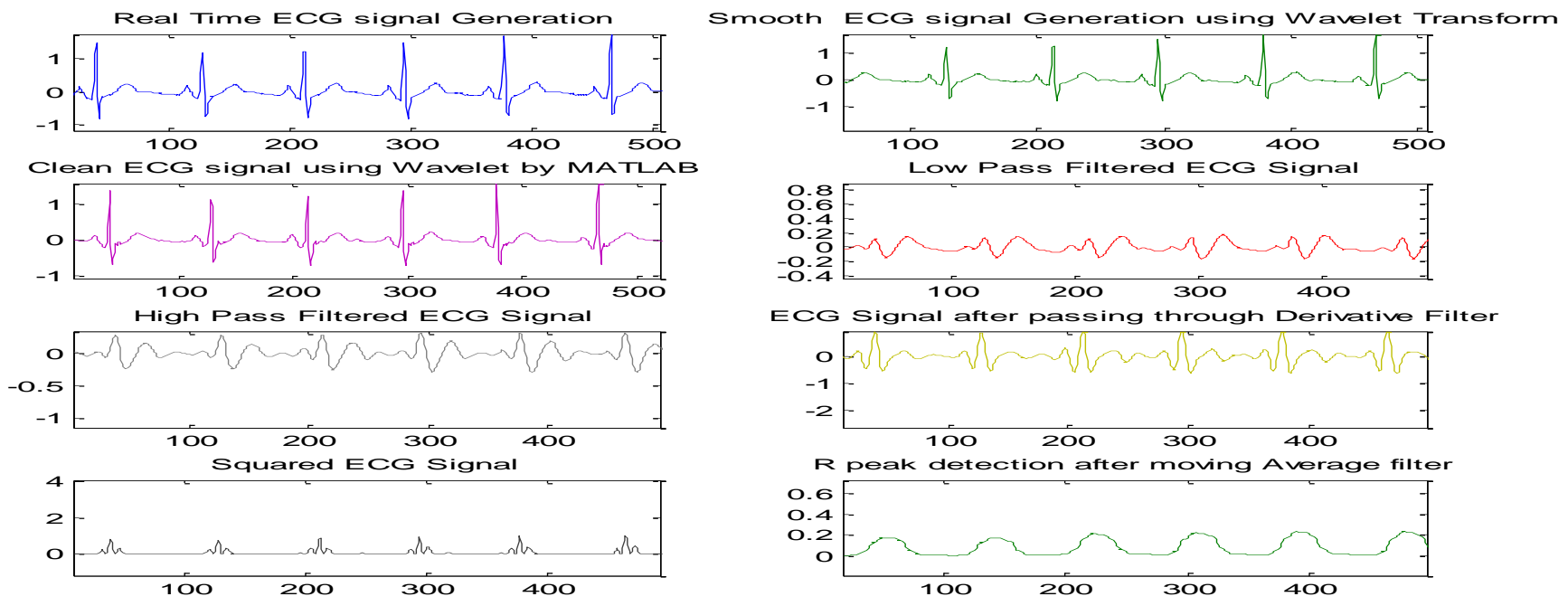

Fig. 4:- implementation of wavelet transform algorithm for r-wave detection

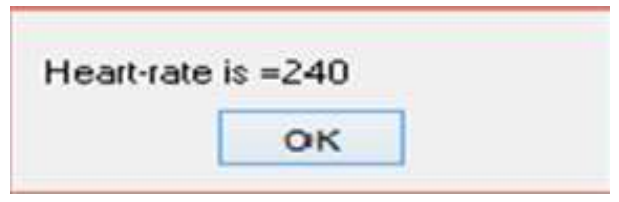

Fig. 5: Heart rate (BPM) calculated from R-R interval 
Table 1 highlights the behaviour of R-wave detection using MATLAB by the implementation of WAVLET TRANSFORM accurately. Here R peak location and its R-R interval are calculated and analyzed.

TABLE 1: R PEAK DETECTION USING MODIFIED WAVELET TRANSFORM

\begin{tabular}{||c|c|c||}
\hline SR \# & R PEAK LOCATION & R-R INTERVAL \\
\hline 1 & 40 & 88 \\
\hline 2 & 128 & 84 \\
\hline 3 & 212 & 83 \\
\hline 4 & 295 & 83 \\
\hline 5 & 378 & 88 \\
\hline 6 & 466 & 93 \\
\hline 7 & 559 & 49 \\
\hline 8 & 608 & 49 \\
\hline 9 & 657 & 93 \\
\hline 10 & 750 & 50 \\
\hline
\end{tabular}

Table 2 highlights the comparison of $\mathrm{R}$ wave extraction with various methods and it is concluded from our results given in table 1, that modified wavelet transform gives us accurate result.

TABLE 2:COMPARISON OF R WAVE EXTRACTION WITH VARIOUS METHODS

\begin{tabular}{|c|c|c|c|c|c|c|}
\hline \multicolumn{7}{|c|}{ Comparison of Algorithms for R wave Extraction using MATLAB } \\
\hline & \multicolumn{2}{|c|}{ Direct Method } & \multicolumn{2}{|c|}{ Pan-Tompkins Method } & \multicolumn{2}{|c|}{ Wavelet Transform Method } \\
\hline Sr\# & $\begin{array}{l}\text { R Peaks } \\
\text { (mSEC) }\end{array}$ & R-R interval & $\begin{array}{l}\text { R Peaks } \\
\text { (mSEC) }\end{array}$ & R-R interval & $\begin{array}{l}\text { R Peaks } \\
\text { (mSEC) }\end{array}$ & $\mathrm{R}-\mathrm{R}$ interval \\
\hline 1 & 40 & 41 & 51 & 89 & 20 & 64 \\
\hline 2 & 81 & 47 & 140 & 84 & 84 & 66 \\
\hline 3 & 128 & 41 & 224 & 83 & 150 & 71 \\
\hline 4 & 169 & 44 & 307 & 83 & 221 & 74 \\
\hline 5 & 213 & 82 & 390 & 87 & 295 & 50 \\
\hline 6 & 295 & 41 & 477 & 50 & 345 & 48 \\
\hline 7 & 336 & 42 & 527 & 44 & 393 & 50 \\
\hline 8 & 378 & 47 & 571 & 50 & 443 & 46 \\
\hline 9 & 425 & 41 & 621 & 47 & 489 & 67 \\
\hline 10 & 466 & 41 & 668 & 50 & 556 & 46 \\
\hline
\end{tabular}

\section{Discussion:}

After using Wavelet transformation, we can easily detect $\mathrm{R}$ wave. R-R interval and heart rate can also be analysed by finding $\mathrm{R}$ wave, from results it is easily concluded that modified wavelet transform gives more accuracy in R-wave detection as revealed from Table 1 and 2 . We can easily identified cardiac diseases after finding heart rate from ECG signal by Equation (5).

\section{Conclusion:}

It is concluded that Wavelet Transformation provide us accurate and efficient result regarding R-peak detection and its results are accurate R-R interval is also calculated form R-Peak detection, algorithm is implemented MATLAB. With this algorithm, abnormalities of the ECG are obtained from the extracted feature. Heart rate is also calculated from R-R intervals and several arrhythmias are also identified from the Heart rate, including Sinus tachycardia, Sinus bradycardia, Supraventricular tachycardia (SVT), Atrial fibrillation (AF), Ventricular tachycardia and Heart block. Hence Wavelet Transform is the best method for the detection of R-Peak from Real time ECG signal generated from MATLAB as it is less time consuming and more efficient.

\section{References}

[1] S. Da, Clinical Electrocardiography. St. Louis, MO, USA: Mosby,1977.

[2] S. Da, Primary Cardiology, G. K. Parisianos, Ed. Belmont, MA, USA: Athena Scientific, 1991.

[3] P. de Chazal, B. G. Celler, R. B. Rei, "Using Wavelet Coefficients for the Classification of the Electrocardiogram", 
Proceedings of the 22nd Annual EMBS International Conference, July 23-28, 2000, Chicago IL.

[4] N. Srinivasan, D. F. Ge, S. M. Krishnan, “Autoregressive Modeling and Classification of Cardiac Arrhythmias”, Proceedings of the Second Joint Conference Houston. TX. USA - October 23-26,2W2

[5] Hafizah Hussain, Lai Len Fatt, "Efficient ECG Signal Classification Using Sparsely Connected Radial Basis Function Neural Network", Proceeding of the 6th WSEAS International Conference on Circuits, Systems, Electronics, Control and Signal Processing, December 2007, pp. 412-416.

[6] [Marcel0 R. Risk, Jamil F. Sobh, J. Philip Saul, "Beat Detection and Classification of ECG Using Self Organizing Maps”, Proceedings - 19th International Conference - IEEEIEMBS, Chicago, IL. USA Oct. 30 - Nov. 2 , 1997

[7] Yuksel Ozbay, Rahime Ceylan, Bekir Karlik, "Integration of type 2 fuzzy clustering and wavelet transform in a neural network based ECG classifier”, Expert Systems with Applications, vol. 38, pp. 1004-1010, 2011.

[8] N. Maglaveras, T. Stamkapoulos, K. Diamantaras, C. Pappas, M. Strintzis, "ECG pattern recognition and classification using nonlinear transformations and neural networks: A review”, Int. J. Med. Inform, vol 52, pp. 191-208, 1998.

[9] X. Liu, Y. J. Zheng, M.W. Phyu, B. Zhao,M. Je, and X. J. Yuan, “A miniature on-chip multi-functional ECG signal processor with $30 \mu \mathrm{W}$ ultralow power consumption,” in Proc. Annu. Int. Conf. IEEE Engineering in Medicine and Biology, Buenos Aires, Argentina, pp. 2577-2580, 2010.

[10] S. Y. Hsu et al., "A sub-100 $\mu \mathrm{W}$ multi-functional cardiac signal processor for mobile healthcare applications," in Proc. Symp. VLSI Circuits, Honolulu, HI, USA, pp. 156-157 2012.

[11] L. F. Cheng, T. C. Chen, and L. G. Chen, "Architecture design of the multifunctional wavelet-based ECG microprocessor for realtime detection of abnormal cardiac events," in Proc. Annu. Int. Conf. IEEE Engineering in Medicine and Biology Soc., San Diego, CA, USA, pp. 4466-44692012.

[12] S. Ari, "ECG signal enhancement using S-Transform," Comput. Biol. Med., vol. 43, no. 6, pp. 649-660, Jul. 2013.

[13] M. Zivanovic and M. Gonz'alez-Izal, "Simultaneous powerline interference and baseline wander removal from ECG and EMG signals by sinusoidal modeling,” Med. Eng. Phys., vol. 35, no. 10, pp. 1431 -1441, Oct. 2013.

[14] Fasano and V. Villani, "Baseline wander removal for bioelectrical signals by quadratic variation reduction," Signal Process, vol. 99, pp. 48 57, Jun. 2014.

[15] M. Merino, "Envelopment filter and K-means for the detection of QRS waveforms in electrocardiogram," Med. Eng. Phys., vol. 37, no. 6, pp. 605-609, Jun. 2015.

[16] C.Ye, "Heartbeat classification using morphological and dynamic features of ECG signals," IEEE Trans. Biomed. Eng., vol. 59, no. 10, pp. 2930-2941, Oct. 2012.

[17] C. A. Bustamante, "ECG delineation and ischemic ST-segment detection based in wavelet transform and support vector machines," in Proc. Pan Amer. Health Care Exchanges, pp. 1-7, 2013.

[18] Y.-C. Yeh, "Feature selection algorithm for ECG signals using range overlaps method," Expert Syst. With Appl., vol. 37, no. 4, pp. 3499-3512, 2010.

[19] L. Mariano and M. J. Pablo, "Heartbeat classification using feature selection driven by database generalization criteria,” IEEE Trans Biomed. Eng., vol. 58, no. 3, pp. 616-625, Mar. 2011.

[20] J. Oster et al., "Semi-supervised ECG ventricular beat classification with novelty detection based on switchingKalman filters," IEEE Trans.Biomed. Eng., vol. 62, no. 9, pp. 2125-2134, Sep. 2015.

[21] L. de Oliveira, "Premature ventricular beat classification using a dynamic Bayesian network," in Proc. IEEE Int. Conf. Eng. Med. Biol. Soc., pp. 4984-4987, Aug./Sep. 2011.

[22] G. de Lannoy, "Weighted conditional random fields for supervised interpatient heartbeat classification," IEEE Trans. Biomed. Eng., vol. 59, no. 1, pp. 241-247, Jan. 2012.

[23] X. Jiang, "ECG arrhythmias recognition system based on independent component analysis feature extraction," in Proc. IEEE Region 10 Conf., pp. 1-4, Nov. 2006.

[24] T. Ince, “A generic and robust system for automated patient-specific classification of ECG signals," IEEE Trans. Biomed. Eng., vol. 56, no. 5, pp. 1415-1426, May 2009.

[25] M. Lagerholm, “Clustering ECG complexes using Hermite functions and self-organizing maps,” IEEE Trans. 
Biomed. Eng., vol. 47, no. 7, pp. 838- 848, Jul. 2000.

[26] V. S. R.Kumari and P. R.Kumar, "Cardiac arrhythmia prediction using improved multilayer perceptron neural network,” Int. J. Electron., Commun, Instrum. Eng. Res. Develop., vol. 3, no. 4, pp. 73-80, 2013.

[27] C. V. Banupriya and S. Karpagavalli, "Electrocardiogram beat classification using probabilistic neural network," Int. J. Comput. Appl. (IJCA), vol. 1, no. 7, pp. 31-37, 2014.

[28] L. S. de Oliveira, "Premature ventricular beat classification using a dynamicBayesian network," in Proc IEEE Eng. Med. Biol Soc. Conf., pp. 4984-4987,2011.

[29] S. Raj, K. Maurya, and K. C. Ray, "A knowledge-based real timeembedded platform for arrhythmia beat classification,” Biomed. Eng.Lett., vol. 5, no. 4, pp. 271-280, Dec. 2015.

[30] F. Melgani and Y. Bazi, "Classification of electrocardiogram signals with support vector machines and particle swarm optimization,” IEEE Trans. Inf. Technol. Biomed., vol. 12, no. 5, pp. 667-677, Sep. 2008.

[31] S. Osowski, L. T. Hoai, and T. Markiewicz, "Support vector machinebased expert system for reliable heartbeat recognition,” IEEE Trans. Biomed. Eng., vol. 51, no. 4, pp. 582-589, Apr. 2004.

[32] S. Raj, S. Luthra, and K. C. Ray, "Development of handheld cardiac event monitoring system," IFACPapersOnLine, vol. 48, no. 4, pp. 71-76, 2015.

[33] C. Ye, B. V. K. V. Kumar, and M. T. Coimbra, "Heartbeat classification using morphological and dynamic features of ECG signals,” IEEE Trans. Biomed. Eng., vol. 59, no. 10, pp. 2930-2941, Oct. 2012.

[34] S. Raj, K. C. Ray, and O. Shankar, "Cardiac arrhythmia beat classification using DOST and PSO tuned SVM," Comput. Methods Programs Biomed., vol. 136, pp. 163-177, Nov. 2016.

[35] K. Minami, H. Nakajima, and T. Toyoshima, "Real-time discrimination of ventricular tachyarrhythmia with Fourier-transform neural network,” IEEE Trans. Biomed. Eng., vol. 46, no. 2, pp. 179-185, Feb. 1999.

[36] T. Ince, S. Kiranyaz, and M. Gabbouj, "A generic and robust system for automated patient-specific classification of ECG signals,” IEEE Trans. Biomed. Eng., vol. 56, no. 5, pp. 1415-1426, May 2009.

[37] S. Banerjee and M. Mitra, "Application of cross wavelet transform for ECG pattern analysis and classification," IEEE Trans. Instrum. Meas., vol. 63, no. 2, pp. 326-333, Feb. 2014.

[38] S. Raj, G. S. S. P. Chand, and K. C. Ray, “Arm-based arrhythmia beat monitoring system,” Microprocess. Microsyst., vol. 39, no. 7, pp. 504-511, 2015.

[39] Akansu,A.N. and Haddad,R.A.,Multiresolution Signal Decomposition: Transforms, subbands, wavelets. Academic Press, San Diego CA,1992.

[40] Patrick S. Hamilton, Willis J. Tompkins, -Quantitative Investigation of QRS Detection rules using the MIT/BIH Arrhythmia Database II , IEEE Transactions on Biomedical Engineering, Vol. BME-33, N0.12, December 1986, PP No. 1157-1165.

[41] ]E.Arrais,Valentim and G.B.Brandao, "Real Time QRS Detection Based on Redundant Discrete Wavelet Transform,” IEEE LATIN AMERICA TRANSACTIONS, VOL. 14, NO. 4, pp. 1662-1668, APRIL 2016. 